\section{Avaliação da cobertura do Serviço Odontológico da Polícia Militar da Bahia em Salvador, Bahia, Brasil}

\author{
Evaluation of dental care coverage in the State \\ Military Police in Salvador, Bahia, Brazil
}

\begin{abstract}
This study seeks to evaluate dental care coverage in the State Military Police in Salvador, Bahia State, Brazil, from 2002 to 2004, estimating potential and real coverage rates. A single descriptive study was performed. Calculations were made of potential coverage rates considering hourly workloads of staff dentists and the real rates resulting from actual outpatient treatment. Potential human resources coverage was adequate (1 dentist per 1,618 policemen), while the real coverage rate was considered below the standard proposed by the Brazilian Ministry of Health (0.39 procedures per policeman per year). The low real coverage rate could be related to low productivity, the reasons for which should be investigated in greater depth in future studies, and might include organizational problems and lack of a management system to improve the quality of professional practice, with specifically defined targets.
\end{abstract}

Oral Health; Health Services Coverage; Police
Clóvis Ribeiro-Sobrinho 1

Luís Eugênio Portela Fernandes de Souza 2

Sônia Cristina Lima Chaves ${ }^{3}$

\section{Introdução}

A cobertura dos serviços de saúde tem sido considerada por vários autores um dos principais atributos para avaliação dos mesmos 1,2,3. No que se refere à saúde bucal, a disponibilidade dos serviços odontológicos na rede do Sistema Único de Saúde (SUS) cresceu substancialmente a partir do final da década de 80 com a municipalização da saúde.

Em 2000, estimava-se que 50\% dos cirurgiões-dentistas brasileiros estariam vinculados ao SUS 4 . Porém, segundo estudo realizado em 2003 pelo Conselho Federal de Odontologia (CFO), apenas $26,2 \%$ dos cirurgiões-dentistas brasileiros teriam vínculo com o setor público 5 . Em 2005, observou-se no Brasil uma relação dentista/habitante de 1/905. Enquanto a menor relação proposta tem sido de um cirurgião-dentista/1.500 habitantes 6,7. Contudo, constatamse diferenças regionais. Em Salvador e São Paulo a relação é de 1/766 e 1/431, respectivamente. Além disso, houve um crescimento de cirurgiões-dentistas neste último ano 3,5 vezes superior à estimativa de crescimento da população brasileira no mesmo período 8,9 , ou seja, há problemas relacionados ao número de cirurgiõesdentista, e também na sua distribuição.

Estudos apontam que a oferta de serviços odontológicos é maior no Sudeste em relação ao Nordeste, sugerindo que o acesso aos serviços odontológicos seguem igual relação 4,10. 
O modelo assistencial privatista predominante no Brasil, aliado à desigualdade sócio-econômica, má distribuição dos profissionais de saúde, baixa capacidade instalada e financiamento irregular do setor, tem levado a uma oferta desigual dos serviços odontológicos, comprometendo assim o acesso aos serviços, especialmente da população menos favorecida sócio-economicamente 11.

Há, portanto, a necessidade de maiores investigações para identificar a cobertura dos serviços odontológicos, no intuito de contribuir para a definição de estratégias para a melhoria do acesso da população a esse bem social.

Em princípio, o acesso deveria ser garantido pelo SUS. Pela dimensão territorial e populacional do Brasil e pela complexidade sócio-política do seu processo de implementação, o SUS tem encontrado, no entanto, dificuldades em tal ampliação. Nesse sentido, os serviços próprios de instituições públicas, como a Polícia Militar da Bahia, poderão contribuir para a melhoria da saúde bucal, prestando atenção odontológica a seus integrantes e dependentes.

A cobertura é um atributo básico na avaliação de um serviço de saúde, pois só se pode avaliar qualquer outro atributo, se houver a oferta e a utilização de um serviço 1 . A cobertura mede a proporção da população que se beneficia do serviço, podendo ser referida como cobertura potencial e cobertura real. A cobertura potencial está relacionada com a oferta de serviços, considerando os recursos materiais ou humanos disponíveis no serviço e mede a quantidade de indivíduos que poderiam ser atendidos, dada a oferta. A cobertura real, por sua vez, está relacionada com a utilização do serviço, correspondendo à proporção da população que efetivamente o utilizou, vale dizer, a taxa de cobertura real equivale à razão entre o número de pessoas que utilizaram o serviço em relação ao público-alvo total 3,12. A cobertura dos serviços de saúde é uma questão importante para o campo da gestão em saúde.

O Serviço Odontológico da Polícia Militar da Bahia (SOPMBA) é constituído de um centro odontológico (Odontoclínica) e consultórios odontológicos em algumas organizações policiais militares (OPM) denominadas de Formação Sanitária, sendo dez em Salvador, duas na sua região metropolitana e nove no interior do Estado da Bahia, com uma concentração da oferta de cirurgiões-dentistas em Salvador de aproximadamente $80 \%$. O SOPMBA, em tese, deve atender a todos os seus integrantes e dependentes, porém há indícios de que o serviço não tem conseguido cumprir tal missão. Assim, este estudo buscou conhecer a cobertura potencial e real do SOPMBA na cidade de Salvador.

\section{Metodologia}

\section{Desenho do estudo}

Esta foi uma pesquisa avaliativa de estudo de caso único, descritivo. O caso foi o SOPMBA na cidade de Salvador, tendo uma população residente em Salvador com direito a utilizar os serviços odontológicos da Polícia Militar da Bahia, somado aos residentes na região metropolitana de Salvador e interior que buscam o SOPMBA da capital.

\section{Técnica de coleta de dados}

Para o cálculo da cobertura do serviço, levantouse a população de policiais militares da ativa e reserva e funcionários civis, residentes em Salvador, entre 2002 e 2004, valendo-se dos dados da Unidade de Pessoal da Polícia Militar Bahia. Para obtenção do número de dependentes, baseouse na fecundidade total no Estado da Bahia de 2,4113 , estimando-se que cada policial tem 3,41 dependentes e somando-se 5.610 pensionistas (incluindo dependentes), a população estimada, com direito a usar o SOPMBA em Salvador chegou a 59.546, 62.045 e 63.120 indivíduos respectivamente nos anos de 2002, 2003 e 2004.

Para o cálculo do numerador da taxa de cobertura real (utilização dos serviços), foi realizado o levantamento do número de procedimentos odontológicos realizados no serviço nos anos de 2002, 2003 e 2004, por intermédio dos mapas de produção de serviços (dados secundários) em todas as unidades da Polícia Militar da Bahia, onde há serviço odontológico em Salvador. Os procedimentos foram classificados em procedimentos coletivos, urgências, diagnósticos clínicos e especializados. Os procedimentos foram classificados em ações básicas correspondendo à somatória dos procedimentos de urgências, diagnósticos e clínicos; e em ações especializadas correspondendo aos procedimentos especializados, segundo padrão proposto pelo Ministério da Saúde 7. Entre as ações básicas, as urgências foram aquelas de drenagem de abscesso, pulpotomia, restauração provisória e cimentação de prótese unitária; as ações de diagnóstico foram a consulta odontológica e as radiografias; os procedimentos clínicos foram as profilaxias, raspagens, aplicações de flúor, selantes, restaurações de amálgama, resina e ionômero, extrações simples de dentes decíduos e permanentes. Dentre as ações especializadas, consideraramse os procedimentos cirúrgicos mais complexos (extração cirúrgica, cirurgias pré-protética, para-endodôntica e muco-gengival, exerese de tumor, frenectomia labial e lingual), procedimentos protéticos (próteses unitárias, fixa, total, 
parcial e adesiva), endodônticos e ortodônticos. Além disso, foram considerados procedimentos coletivos: palestras, bochechos com flúor e escovação orientada com flúor.

A oferta potencial do serviço foi estimada pelo número de cirurgiões-dentistas existentes nos anos de 2002, 2003 e 2004, identificados mediante relatórios e documentos. O padrão utilizado para o cálculo da cobertura potencial de procedimentos foi aquele proposto pelo Ministério da Saúde, na Portaria $n^{\circ}$. 1.101, de 2002, na relação entre cirurgiões-dentistas e a capacidade de atendimento, correspondendo a três consultas ou procedimentos/hora e dois procedimentos/ habitante/ano, para identificar o numerador da taxa de cobertura potencial 7. Foi utilizado um padrão de 1,6 procedimento básico/habitante/ ano e 0,4 procedimento especializado/habitante/ano, partindo-se do pressuposto de que a soma de ambos atingiria o padrão de 2 procedimentos odontológicos/habitantes/ano.

\section{Análise dos dados}

Foram obtidas a relação e a proporção da cobertura potencial referente aos recursos humanos (cirurgiões-dentistas) do SOPMBA na Bahia e na cidade de Salvador, como também a oferta, a taxa e a proporção da cobertura potencial, referente aos procedimentos do SOPMBA na cidade de Salvador nos anos de 2002, 2003 e 2004.

A cobertura real foi analisada segundo os procedimentos básicos (urgência, diagnóstico e clínico) e especializados, obtendo-se a oferta, a taxa de cobertura e a proporção da cobertura reais tanto dos procedimentos básicos como dos especializados. Os dados foram digitados e analisados em tabelas dinâmicas do programa Excel (Microsoft Corp., Estados Unidos).

\section{Resultados}

Cobertura potencial do SOPMBA, considerando a quantidade de cirurgiões-dentistas

No SOPMBA havia 49 cirurgiões-dentistas em 2002, 54 em 2003 e 55 em 2004. Destes, 5 (10,2\%) em 2002, 5 (9,3\%) em 2003 e 6 (10,9\%) em 2004 encontravam-se em função administrativa, realizando curso ou à disposição. Restando 44 cirurgiões-dentistas em atividade no ano de $2002 \mathrm{e}$ 49 cirurgiões-dentistas em 2003 e 2004. A relação cirurgião-dentista/habitante foi de 2.880, 2.713 e 2.773, respectivamente nos anos de 2002, 2003 e 2004. Tais relações estão acima da relação cirurgião-dentista/habitante do Brasil (1/936) e da
Bahia (1/2.357) em 2004 5. Alguns documentos recomendam o padrão de 1 cirurgião-dentista para 1.500 habitantes 6,7. Logo, a relação cirurgião-dentista/habitante no SOPMBA em todo o Estado da Bahia atingiu apenas 52\%, 55\% e 54\% do padrão proposto, respectivamente em 2002, 2003 e 2004. Além disso, cabe destacar que a nãocontratação de novos profissionais desde 1995 poderá agravar a baixa cobertura potencial de recursos humanos encontrada em odontologia (Tabela 1).

Cumpre destacar que $80 \%$ dos cirurgiõesdentistas do SOPMBA trabalhavam em Salvador, dos quais $72 \%$ no centro odontológico especializado em diversas especialidades odontológicas, incluindo prótese e ortodontia. Os demais trabalhavam em unidades independentes. Apenas dez cirurgiões-dentistas (20\%) trabalhavam em unidades no interior do Estado da Bahia e dentre eles, dois na região metropolitana de Salvador. Assim, a relação cirurgião-dentista/habitante no SOPMBA em Salvador foi de 1/1.751, 1/1.551 e $1 / 1.618$, atingindo $85,7 \%, 96,7 \%$ e $92,7 \%$ do padrão proposto, respectivamente em 2002, 2003 e 2004.

Os serviços especializados são referenciados apenas para o único centro odontológico (Odontoclínica) em Salvador, o que já caracteriza uma barreira de acesso organizacional e geográfica importante.

\section{Cobertura potencial do atendimento odontológico em Salvador}

A cobertura potencial do SOPMBA em Salvador foi calculada considerando o padrão do SUS, que é de 3 procedimentos/cirurgião-dentista/hora. Considerando 16 horas semanais de trabalho por cirurgião-dentista do SOPMBA, quatro semanas no mês e 11 meses no ano, a oferta potencial de serviço totalizou 2.112 procedimentos/cirurgião dentista por ano. Assim em 2002, com 34 cirurgiões-dentistas em atividade, houve uma oferta de 71.808 procedimentos para uma população estimada de 59.546 indivíduos, resultando numa taxa de cobertura potencial de 1,21 procedimentos/habitante/ano, correspondendo a $60,5 \%$ do padrão proposto de 2 procedimentos/habitante/ano (Tabela 1).

Em 2003, com 39 cirurgiões dentistas em atividade, ocorreu uma oferta de 82.368 procedimentos, para uma população estimada de 62.053 indivíduos, resultando em uma taxa cobertura potencial de 1,36 procedimentos/habitante/ano, atingindo $68 \%$ do padrão proposto (Tabela 1).

Em 2004, com 40 cirurgiões-dentistas em atividade a oferta foi de 84.480 procedimentos, para uma população estimada de 63.120 indivíduos, 
Cobertura potencial e real e procedimentos em odontologia com as respectivas taxas do Serviço Odontológico da Polícia Militar da Bahia no período entre 2002 e 2004. Salvador, Bahia, Brasil.

\begin{tabular}{|c|c|c|c|}
\hline Indicadores & 2002 & 2003 & 2004 \\
\hline População-alvo estimada na Bahia & 126.704 & 132.938 & 135.870 \\
\hline Relação cirurgião-dentista/habitante na Bahia & $1 / 2.880$ & $1 / 2.713$ & $1 / 2.773$ \\
\hline Oferta potencial de recursos humanos na Bahia & $52,1 \%$ & $55,3 \%$ & $54,1 \%$ \\
\hline População-alvo estimada em Salvador & 59.546 & 62.053 & 63.120 \\
\hline Relação cirurgião-dentista/habitante em Salvador & $1 / 1.751$ & $1 / 1.551$ & $1 / 1.618$ \\
\hline Cobertura potencial de recursos humanos em Salvador & $85,7 \%$ & $96,7 \%$ & $92,7 \%$ \\
\hline Oferta potencial de procedimentos & 71.808 & 79.194 & 82.368 \\
\hline Taxa de cobertura potencial (consulta ou procedimento/habitante/ano) & 1,21 & 1,36 & 1,30 \\
\hline Cobertura potencial de procedimentos & $60,5 \%$ & $68,0 \%$ & $65,0 \%$ \\
\hline Oferta real de ações básicas & 23.400 & 23.201 & 24.387 \\
\hline Taxa de cobertura real (ações básicas/habitante/ano) & 0,39 & 0,37 & 0,39 \\
\hline Cobertura real de ações básicas & $24,4 \%$ & $23,1 \%$ & $24,4 \%$ \\
\hline Oferta real de procedimentos especializados & 5.896 & 7.520 & 8.187 \\
\hline Taxa de cobertura real (procedimento especializado/habitante/ano) & 0,10 & 0,12 & 0,13 \\
\hline Cobertura real de ações especializadas & $24,8 \%$ & $30,3 \%$ & $32,4 \%$ \\
\hline
\end{tabular}

resultando em uma taxa cobertura potencial de 1,30 procedimentos/habitante/ano, correspondendo a $65 \%$ do padrão proposto (Tabela 1 ).

Cobertura real do atendimento odontológico

Em 2002, o SOPMBA em Salvador realizou 23.400 procedimentos de ação básica e 5.896 procedimentos especializados, para uma população estimada de 59.546 indivíduos, resultando em uma taxa de cobertura real de ações básicas de 0,39 procedimentos/habitante/ano. A taxa de cobertura real de ações especializadas foi de 0,10 procedimento/habitante/ano em 2002 (Tabela 1).

Em 2003, o SOPMBA em Salvador produziu 23.201 ações básicas em odontologia e 7.520 procedimentos especializados para uma população estimada de 62.053 indivíduos, resultando em uma taxa de cobertura real de ações básicas de 0,37 procedimento/habitante/ano. A taxa de cobertura real de ações especializadas foi de 0,12 procedimento/habitante/ano em 2003 (Tabela 1).

Em 2004, o SOPMBA em Salvador produziu 24.387 ações básicas em odontologia e 8.187 procedimentos especializados, para uma população estimada de 63.120 indivíduos, resultando em uma taxa de cobertura real de ações básicas de 0,39 procedimento/habitante/ano. A taxa de cobertura real de ações especializadas foi de 0,13 procedimento/habitante/ano em 2004 (Tabela 1).
O padrão proposto pelo Ministério da Saúde é de 0,4 a 1,6 procedimento de ação básica em odontologia/habitante/ano, e de 0,04 a 0,06 procedimento especializado/habitante/ano ${ }^{7}$. No entanto, para atender o padrão proposto de 2 procedimentos/habitante/ano, utilizou-se o padrão de 1,6 procedimento de ação básica em odontologia/habitante/ano e 0,4 procedimento odontológico especializado/habitante/ano. Observou-se, portanto, que em nenhum dos anos avaliados, o SOPMBA em Salvador atingiu o padrão proposto. Apenas os procedimentos de ações básicas em odontologia se aproximaram do padrão mínimo sugerido, enquanto os procedimentos especializados encontravam-se muito baixo do proposto. Isso pode estar relacionado a pouca oferta desses procedimentos na única unidade de referência para procedimentos especializados do SOPMBA em todo o Estado da Bahia (Figura 1).

Verificou-se também que a taxa de cobertura real correspondeu a apenas 40,8\% em 2002, $36,4 \%$ em 2003 e 39,5\% em 2004, da taxa de cobertura potencial, respectivamente. Como também chamou a atenção que a proporção da cobertura real de procedimentos básicos foi de apenas $24,4 \%, 23,1 \%$ e $24,4 \%$, em relação ao padrão de 1,6 procedimentos/habitante/ano e a proporção da cobertura real de procedimentos especializados foi de apenas $24,8 \%, 30,3 \% \mathrm{e}$ $32,4 \%$ em relação ao padrão de 0,4 procedimento/habitante/ano, respectivamente entre $2002 \mathrm{e}$ 2004 (Tabela 1; Figura 2). 
Taxa de cobertura potencial e real de procedimentos do Serviço Odontológico da Polícia Militar da Bahia nos anos de 2002, 2003 e 2004. Salvador, Bahia, Brasil.

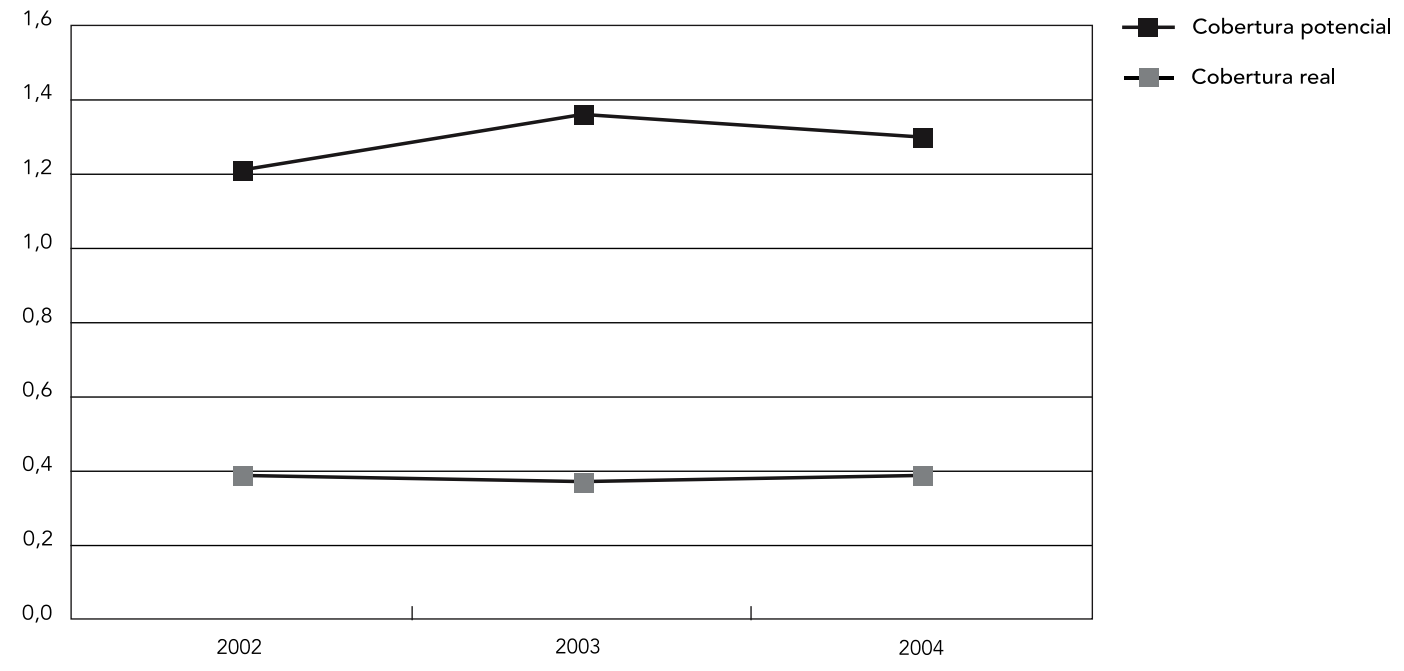

Além disso, caberia destacar a baixa proporção de procedimentos coletivos em saúde bucal no SOPMBA em relação ao total de ações desenvolvidas, em 2002 (4,7\%), 2003 (1,5\%) e 2004 $(3,4 \%)$, caracterizando a ênfase do serviço em ações curativas e individuais em detrimento de práticas preventivo-educativas de base populacional. Apesar disso, o serviço não apresentou uma característica mutiladora, já que as proporções de exodontias em relação às ações odontológicas básicas individuais na Odontoclínica foram de $3 \%, 2,7 \%$ e $3,2 \%$, respectivamente, entre os anos de 2002 a 2004 (Tabela 2).

\section{Discussão}

O estudo revelou uma baixa cobertura potencial na relação cirurgião-dentista/habitante em toda Polícia Militar da Bahia. Contudo, tal relação, em Salvador, esteve próxima ao padrão proposto, pelo fato de aproximadamente $80 \%$ dos dentistas da Polícia Militar da Bahia estarem lotados em Salvador. A relação cirurgião-dentista/habitante do SOPMBA em Salvador assemelha-se à encontrada em cidades do Estado de Santa Catarina, que, conforme estudo em 293 municípios, aponta para a média de 1 cirurgião-dentista/ 1.830 habitantes 14. Entretanto tal relação cirurgião-dentista/habitante em Salvador foi de 1/2.941 15,16.
A oferta potencial do SOPMBA de procedimentos só atingiu, em média, $65 \%$ do padrão sugerido. Uma das razões da baixa oferta potencial pode ser a carga horária semanal de 16 horas, uma vez que o padrão proposto pelo SUS é de 20 horas, o que levaria a uma cobertura potencial de $82 \%$.

De fato, só um cirurgião-dentista do SOPMBA em Salvador relatou trabalhar 20 horas semanais, ou seja, cinco turnos por semana. Todavia, com base nos mapas de produção do serviço, constatou-se que a média real de turnos trabalhados/ semana/cirurgião-dentista foi de 2,6 (2002), 2,7 (2003) e 3,0 (2004). Essa defasagem possivelmente pode ser explicada por feriados, dispensas diversas, problemas de gestão, equipamentos e falta de material.

Tal situação pode estar relacionada à baixa proporção de cobertura real do SOPMBA em Salvador (24\%) e aquém da cobertura potencial dos procedimentos odontológicos. A taxa de cobertura real de ações básicas de odontologia/habitante/ano foi de 0,39 em 2004, enquanto tal taxa na Bahia, de procedimentos produzidos pelo SUS, foi de 0,60. Em São Paulo a taxa foi de 1,37 e em Salvador de 0,42 15,17,18. A taxa de cobertura real de procedimentos especializados em odontologia do SOPMBA em 2004 foi de 0,13 , atingindo apenas $32,5 \%$ do padrão mínimo proposto. Tal taxa supera, porém, as mesmas da 
Figura 2

Percentual da cobertura potencial e real no Serviço Odontológico da Polícia Militar da Bahia, nos anos de 2002 , 2003 e 2004 . Salvador, Bahia.

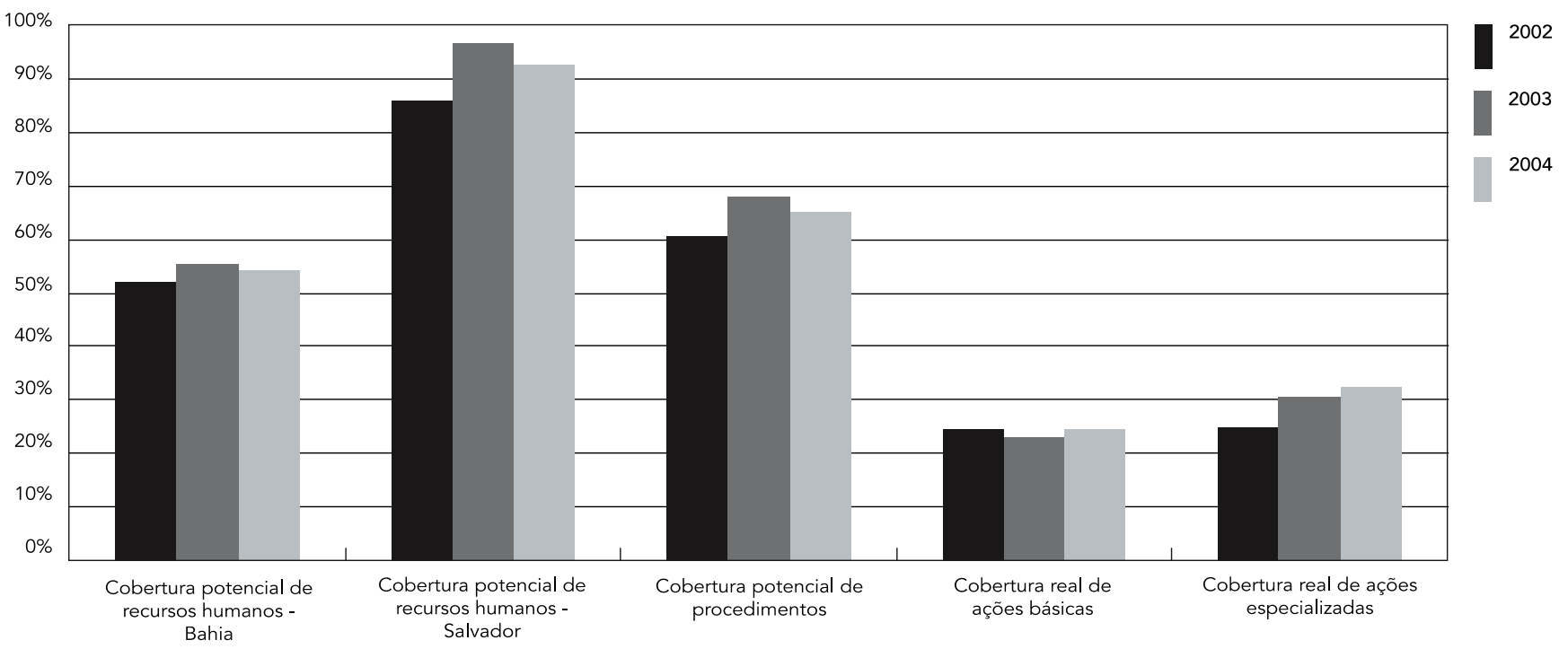

Tabela 2

Proporção de procedimentos coletivos e exodontias em relação às ações básicas em odontologia no Serviço Odontológico da Polícia Militar da Bahia, no período entre 2002 e 2004. Salvador, Bahia, Brasil.

\begin{tabular}{lccc}
\hline Indicadores & $\mathbf{2 0 0 2}$ & $\mathbf{2 0 0 3}$ & $\mathbf{2 0 0 4}$ \\
\hline Procedimentos coletivos & $4,7 \%$ & $1,5 \%$ & $3,4 \%$ \\
Proporção de exodontias & $3,0 \%$ & $2,7 \%$ & $3,2 \%$ \\
\hline
\end{tabular}

Bahia (0,02), São Paulo $(0,05)$ e Salvador $(0,05)$ no SUS. Confirmou-se, por conseguinte, a baixa cobertura dos serviços públicos odontológicos especializados e a má distribuição deles, 4,19,20, comprometendo o princípio de integralidade. Além disso, a baixa proporção de procedimentos coletivos na prevenção de doenças bucais realizadas pelo serviço pode potencializar o aumento das necessidades e, conseqüentemente, a sua demanda. Com isso, agravam-se os principais problemas encontrados no SOPMBA como a baixa cobertura potencial e real.

O SOPMBA, em 2004, apresentou uma relação cirurgião-dentista/habitante mais favorável que a encontrada na Bahia. Assim, esperava-se que a taxa de cobertura real fosse superior no
SOPMBA em relação à Bahia, já que uma maior oferta poderia proporcionar um maior acesso e utilização do serviço, fato que não se confirmou. Portanto, talvez haja sérios problemas de acesso e utilização por parte dos usuários do serviço odontológico da Polícia Militar da Bahia, merecendo estudos de avaliação da acessibilidade do serviço e intervenções voltadas para seu aumento, conforme apontam alguns autores 21,22,23, a fim de contribuir para a melhoria do serviço. Importante salientar que o SOPMBA, apesar da baixa cobertura real de procedimentos básicos, apresentou uma expressiva cobertura real de procedimentos especializados em relação ao SUS, destacando que os praças (sargentos e soldados), representam $85 \%$ do público alvo do serviço, os quais seriam potencialmente usuários do SUS. Também os serviços odontológicos da Marinha, Exército e Aeronáutica existentes em Salvador têm centros odontológicos especializados. Desse modo, os serviços odontológicos militares contribuem para a universalização do SUS, sendo mais atuantes quanto ao princípio de integralidade.

O método utilizado nesta pesquisa de avaliação da cobertura por procedimentos odontológicos realizados ou potenciais, poderá ser um instrumento de avaliação dos serviços odontológicos prestado pelo SUS, em razão de informar com mais precisão tanto o potencial de uti- 
lização do recurso como a efetiva utilização. Tal estratégia também possibilita diferenciar um serviço que atenda prioritariamente à demanda espontânea, por motivo de dor, de outro que busque um atendimento integral ao paciente. A cobertura da primeira consulta odontológica para urgência pode indicar que o serviço prio- ritariamente volta-se à demanda espontânea. Contudo, serviços onde há menor cobertura de consultas de urgência podem estar indicando uma maior ênfase ao atendimento integral das necessidades dos usuários. Sendo assim, espera-se que este método de avaliação da cobertura possa ser utilizado pelos serviços de saúde.

\section{Resumo}

Este estudo visou avaliar a cobertura do Serviço Odontológico da Polícia Militar da Bahia (SOPMBA) na cidade de Salvador, Bahia, Brasil, entre 2002 e 2004, e estimar as taxas de sua cobertura potencial e real. Fo realizado um estudo de caso único, descritivo. Foram calculadas as taxas de cobertura potencial levando-se em conta a carga horária dos cirurgiões-dentistas do serviço e cobertura real, com base na produção ambulatorial odontológica. Encontrou-se uma adequada cobertura potencial de recursos humanos (1 cirurgiãodentista/1.618 habitantes) e a taxa de cobertura real foi considerada abaixo do padrão proposto pelo Ministério da Saúde (0,39 procedimento/habitante/ano). A baixa cobertura real encontrada pode estar relacionada à baixa produtividade do serviço, cujas razões devem ser mais bem investigadas em estudos posteriores como problemas estruturais e a falta de um sistema de gestão do desempenho da qualidade da prática profissional com metas a serem cumpridas.

Saúde Bucal; Cobertura de Serviços de Saúde; Polícia

\section{Colaboradores}

C. Ribeiro-Sobrinho realizou a construção teórico-metodológica da investigação, elaborou os gráficos, analisou os dados e redigiu o texto. L. E. P. F. Souza orientou a condução dos trabalhos e contribuiu na construção teórico-metodológica da investigação. S. C. L. Chaves colaborou na construção teórica da investigação, contribui na análise dos dados e estruturação e revisão do texto final. 


\section{Referências}

1. Formigli VLA, Costa COM, Porto LA. Avaliação de um serviço de atenção integral à saúde do adolescente. Cad Saúde Pública 2000; 16:831-41.

2. Vieira-da-Silva ML, Formigli VLA. Avaliação em saúde: limites e perspectivas. Cad Saúde Pública 1994; 10:80-91.

3. Vieira-da-Silva ML. Conceitos, abordagens e estratégias para a avaliação em saúde. In: Hartz ZMA, Vieira-da-Silva ML, organizadoras. Avaliação em saúde: dos modelos teóricos à prática na avaliação de programas e sistemas de saúde. Salvador: EDUFBA/Rio de Janeiro: Editora Fiocruz; 2005. p. 15-39.

4. Roncalli AG. A organização da demanda em serviços públicos de saúde bucal: universalidade, eqüidade e integralidade em saúde bucal coletiva [Tese de Doutorado]. Araçatuba: Universidade Estadual Paulista; 2000.

5. Conselho Federal de Odontologia. Perfil do cirurgião-dentista no Brasil. http://www.cfo.org. br/download/pdf/perfil_CD.pdf (acessado em 05/ Jan/2006).

6. Queluz DP. Recursos humanos na área odontológica. In: Pereira AC, organizador. Odontologia em saúde coletiva: planejando ações e promovendo saúde. Porto Alegre: Editora Artmed; 2003. p. 14059.

7. Brasil. Portaria n ${ }^{\circ} .1101$. Estabelece os parâmetros de cobertura assistencial conforme deliberação $n^{\circ}$. 8 de 04 de abril de 2002 do Conselho Nacional de Saúde. Diário Oficial da União 2002; 13 jun.

8. Instituto Brasileiro de Geografia e Estatística. Estimativa da população brasileira 2005. http://www. ibge.gov.br/home/estatistica/populacao/default. shtm (acessado em 07/Ago/2005).

9. Conselho Federal de Odontologia. Estatística. http://www.cfo.org.br/numero (acessado em 06/ Out/2005).

10. Ministério da Saúde. Projeto SB Brasil: condições de saúde bucal da população brasileira 2002-2003. http://planeta.terra.com.br/saude/angelonline/ front.htm (acessado em 10/Jul/2004).

11. Zenetti CHG. As marcas do mal-estar social no sistema nacional de saúde: o caso das políticas de saúde bucal, no Brasil dos anos 80 [Dissertação de Mestrado]. Rio de Janeiro: Escola Nacional de Saúde Pública, Fundação Oswaldo Cruz; 1993.
12. Soberon G. La extensión de cobertura de los servicios de salud. Gac Méd Méx 1998; 124:163-75.

13. Instituto Brasileiro de Geografia e Estatística. Estimativa da população brasileira 2004. http://www. ibge.gov.br/home/estatistica/populacao/default. shtm (acessado em 15/Out/2004).

14. Fernandes LS, Peres MA. Associação entre atenção básica em saúde bucal e indicadores sócioeconômicos municipais. Rev Saúde Pública 2005; 39:930-6.

15. Ministério da Saúde. DATASUS: cadernos de informações. Município: Salvador - BA. http://tabnet. datasus.gov.br/tabdata/cadernos/BA/BA_Salva dor_Geral.xls (acessado em 05/Jan/2006).

16. Instituto Brasileiro de Geografia e Estatística. Estatística da saúde assistência médico-sanitária 2002. http://www.ibge.gov.br/home/estatistica/popula cao/condicaodevida/ams/default.shtm (acessado em 11/Out/2004).

17. Ministério da Saúde. DATASUS: cadernos de informações gerais. Unidade da Federação: Bahia. http://tabnet.datasus.gov.br/tabdata/cadernos/ BA/BA_Bahia_GeralUF.xls (acessado em 08/ Jan/2006).

18. Ministério da Saúde do Brasil. DATASUS: cadernos de informações. Unidade da Federação: São Paulo. http://tabnet.datasus.gov.br/tabdata/cadernos/ SP/SP_Sao_Paulo_GeralUF.xls (acessado em 08/ Jan/2006).

19. Travassos C. Acesso e utilização de serviços de saúde primeiros resultados do suplemento saúde da PNAD 2003. http://www.cict.fiocruz.br/ arqnoticias/arq2005/pnad.pdf (acessado em 12/ Out/2005).

20. Ministério da Saúde. IDB 2003 Brasil: indicadores de recursos e cobertura. Brasília: Ministério da Saúde; 2003.

21. Frenk JM. El concepto y la medición de accesibilidad. In: White KL, editor. Investigaciones sobre servicios de salud: una antología. Washington DC: Organización Panamericana de la Salud; 1992. p. 929-43.

22. Andersen RM. Revisiting the behavioral model and access to medical care: does it matter? J Health Soc Behav 1995; 36:1-10.

23. Barros AJD, Bertoldi AD. Desigualdades na utilização e no acesso a serviços odontológicos: uma avaliação em nível nacional. Ciênc Saúde Coletiva 2002; 7:709-17.

Recebido em 31/Jan/2007

Versão final reapresentada em 05/Jul/2007

Aprovado em 24/Jul/2007 